\title{
Baby steps towards gene therapy for humans
}

If we were more like mice, we'd probably be a whole lot healthier. Over the past few years, scientists have used gene therapy to treat mice for almost every condition imaginable. Unfortunately, attempts to translate such results into clinical therapy have had limited success, in part because humans are so much larger than mice. Now, Dongsheng Duan (University of Missouri, Columbia) and colleagues have found a way to administer whole-body gene therapy to dogs, suggesting that similar methods may one day be feasible in other large animals such as humans.

Duan's lab investigates Duchenne's muscular dystrophy, a fatal condition caused by mutations in the gene encoding dystrophin. Patients begin to lose motor ability during early childhood, and most die before age 30 . Because all muscles in the body are affected by Duchenne's, gene therapy for such a disease would require whole-body systemic transduction. Recent studies have shown that such treatment is possible in mice, but attempts to use similar techniques in adult dogs have thus far been unsuccessful because of dogs' strong cellular immune response.

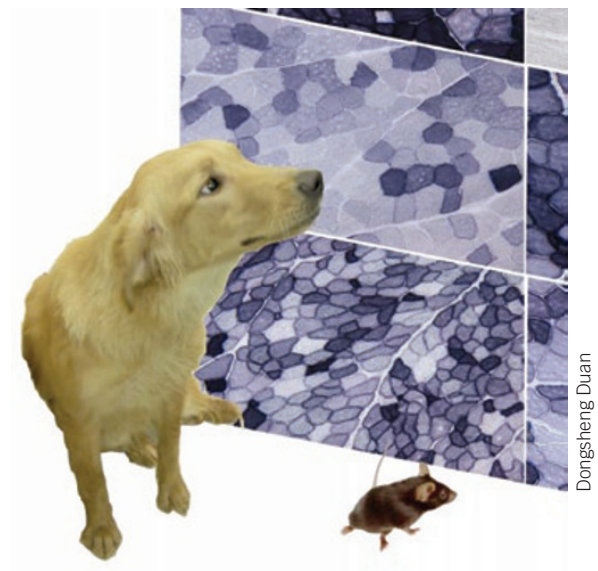

The researchers used two approaches to carry out gene transfer in beagles and in beagle-golden retriever mixes. First, they injected young adult dogs intravenously with a viral vector that had not previously been used in dogs (adeno-associated viral vector serotype 9). The vector, which carried a reporter gene but no therapeutic components, triggered an immune response and yielded limited transduction. The researchers then attempted the same technique in newborn puppies whose immune systems were not yet developed. In this case, skeletal muscle transduction was completely effective and was maintained for 6 months (Mol. Ther. published online 30 September 2008; doi:10.1038/mt.2008.207). In dogs, the transduction qualities of the viral vector were completely different from those previously observed in mice, emphasizing the difficulty in extrapolating results from one species to another.

The team's research shows that administering gene therapy to neonates has a clear immunological advantage, but there may also be a clinical advantage to treatment at a young age. New techniques have been developed to diagnose diseases such as Duchenne's in newborns. If babies can be treated for these conditions before symptoms occur, there is a possibility of avoiding clinical damage altogether.

It remains to be determined whether such gene therapy would be effective in humans. But the research of Duan and colleagues may have brought us one step closer to doing so. Karen Marron

\section{FISHING FOR TOXIC BACTERIA}

Bacterial pathogens in food and water cause more than 76 million infections per year in the US, which have symptoms ranging from mild gastrointestinal discomfort to severe illness and even death. Accurate detection of these pathogens is essential for avoiding infection. Although molecular and immunological detection methods have progressed, they do sometimes fail to identify toxic bacteria: those that rely on specific known DNA or protein sequences may not detect newly emergent or mutated bacteria. At the other end of the spectrum, existing detection methods may also give false positive results, prompting costly recalls and public distress. This is because the techniques cannot distinguish between live versus dead bacteria and cannot directly evaluate toxicity, only bacterial presence. In addition, many current approaches are time-intensive and expensive.

Now, a group of scientists led by Janine Trempy (Oregon State University, Corvallis) has identified a cell-based 'biosensor' that can detect toxins from foodborne bacterial pathogens. The new technology in food safety testing uses pigment-bearing cells called erythropores from Siamese fighting fish (Betta slendens). In response to potentially harmful environmental conditions, including bacterial toxins, the pigment in erythropores redistributes in a characteristic pattern. The pigment shift is quick, can be easily observed using low-power microscopy and can be quantified to describe the intensity of the stimulus (Microb. Biotechnol. 1, 425-431; 2008).

Trempy et al. first tested erythropore response to three types of Bacillus bacteria: $B$. cereus, which can cause vomiting and diarrhea; a $B$. cereus variant engineered not to produce toxin; and $B$. subtilis, which is not pathogenic. They observed the pigment shift only after exposing erythropores to toxic $B$. cereus. The group next assessed whether erythropores responded similarly to other bacterial pathogens: Clostridium perfringens, Clostridium botulinum and Salmonella enteridis. In each case, they observed a pigment response after exposing erythropores to the pathogens but not after exposing them to culture media alone (as a negative control).

More studies are needed before the erythropore method can be used commercially, including an assessment of its ability to detect other potentially toxic bacteria, such as E. coli 0157:H7 and Listeria. But the technology holds promise for improving food safety testing and effectively protecting consumers from foodborne bacterial infections. "When this new technology is commercially available, we should be able to provide a higher level of assurance to the consumer while avoiding the waste of millions of dollars worth of food that is suspected of bacterial contamination, but actually is safe," said Trempy. Monica Harrington 